\title{
Relationships between urine albumin excretion and dietary habits in patients with type
}

\section{2 diabetes}

Sadako Matsui ${ }^{*}$, Yasuhisa Someya ${ }^{2,3}$, Hiroshi Yoshida ${ }^{*}$

1 Food and Nutrition, Faculty of Human Sciences and design, Japan Women's University; smatsui@fc.jwu.ac.jp

2 Someya Clinic; someyac@kch.biglobe.ne.jp

3 Division of Diabetes, Metabolism and Endocrinology, Department of Internal Medicine, The Jikei University School of Medicine.

4 Department of Laboratory Medicine, The Jikei University Kashiwa Hospital; hyoshida@jikei.ac.jp

* Correspondence: smatsui@fc.jwu.ac.jp; Tel.: +81-3-5981-3432 (S.M.); hyoshida@jikei.ac.jp; Tel.: +81-4-71641111/Fax.: +81-4-7166-9374 (H.Y.)

\begin{abstract}
Background: The dietary factors and nutrients contributing to the prevention of microalbuminuria in type 2 diabetic nephropathy is unclear, so we investigated dietary factors affecting urinary albumin excretion in patients with type 2 diabetes. Methods: 42 patients with type 2 diabetes were participated, the subjects were divided to a normal albuminuria group (urinary albumin / creatinine ratio of less than $30 \mathrm{mg} / \mathrm{g} \mathrm{Cr}$ ) and a microalbuminuria group of $30 \mathrm{mg} / \mathrm{g}$ to $299 \mathrm{mg} / \mathrm{g}$ Cr. We performed casual blood sampling and conducted a food frequency questionnaire survey. Results: There were no significant differences in age, BMI and other physiological and biochemical data, the average daily intake of energy and many of nutrients, while $\beta$-cryptoxanthin was significantly lower in the microalbuminuria group than in the normal group (506.4 \pm 793.9 $\mu \mathrm{g} /$ day vs. $715.3 \pm 500.3 \mu \mathrm{g} /$ day, $\mathrm{p}<0.05)$. The intake of 17 food groups per day showed that the intakes of fruits were significantly lower in the microalbuminuria group than in the normal group $(76.9 \pm 134.1 \mathrm{~g}$ vs. $111.9 \pm 84.5 \mathrm{~g}, \mathrm{p}<0.05)$. Conclusion: These results suggest that fruits and foods rich in $\beta$-cryptoxanthin would make it possible to prevent diabetic nephropathy progression.
\end{abstract}

Keywords: Type 2 diabetes; urea albumin excretion; food frequency questionnaire survey; $\beta$ cryptoxanthin; fruits

\section{Introduction}

Diabetic nephropathy is one of the major complications of diabetes as well as retinopathy and neuropathy, and it is representative of diabetic microangiopathy. In Japan, diabetic nephropathy has become first in causes of chronic dialysis therapy since 1998, and it accounted for $43.2 \%$ of total dialysis therapy in 2016 [1]. In diabetic patients, the most influential complication for prognosis is cardiovascular disease, and diabetes patients are more at risk of developing cardiovascular disease than non-diabetic patients [2]. Furthermore, the decline in renal function is associated with cardiovascular disease mortality [3]. As a result, diabetic nephropathy is considered to provide a poor prognosis for life. In addition, due to the sustained hyperglycemia, vascular disorders are progressing, and it would be difficult to carry out dialysis therapy over a long period of time. In fact, it has been reported that the most of leading cause of death was cardiac failure (26.8\%) among Japanese dialysis patients in 2013 [4].

Most of the glomerular filtered proteins are reabsorbed in the proximal tubule. However, when hyperglycemia persists, renal glomerular basement membrane is destroyed, and proteins leak into urine, of which 30 to $40 \%$ are albumin. Albumin escapes into urine as the glomerular barrier 
function declines, and when it comes to microalbuminuria (30 to $299 \mathrm{mg}$ / g Cr), it is diagnosed as diabetic nephropathy (II-stage; early kidney disease) [5]. The presence of microalbuminuria is a risk factor for cardiovascular disease independent of renal dysfunction, and it has been reported that the onset of cardiovascular disease is suppressed when albumin excretion is decreasing [6]. Also, the estimated glomerular filtration rate (eGFR), which is one of the evaluation indices of renal function, is still no less than $60 \mathrm{~mL} / \mathrm{min} / 1.73 \mathrm{~m} 2$ at the second stage of diabetic nephropathy and shows normal to mildly impaired renal function [7], and consequently the measurement of urinary albumin is considered essential for early diagnosis of diabetic nephropathy. These findings indicate that the early detection of diabetic nephropathy prevents the progression of renal dysfunction, launching of dialysis therapy and the onset of cardiovascular disease.

Presently, dietary therapy, which includes some restrictions of dietary protein and sodium, is used widely as a treatment for diabetic nephropathy. In diabetic patients, the dietary protein restriction is an effective treatment for suppressing the progression of renal dysfunction, leading to the reduction of urinary protein $[8,9]$. In addition, since the excess intake of sodium potentially leads to hypertension and subsequent renal vascular injuries, dietary sodium restriction less than 6 $\mathrm{g} /$ day is also recommended to diabetic patients with hypertension [10]. Thus, there are many papers on relations between specific nutrients and renal function, but dietary factors and nutrients contributing to the prevention of microalbuminuria in type 2 diabetic nephropathy are not well defined. Therefore, we investigated dietary factors affecting the urinary albumin excretion in terms of dietary intake situations in patients with type 2 diabetes.

\section{Materials and Methods}

Forty-two patients with type 2 diabetes (29 men and 13 women) participated in the present study, who visited Someya outpatient clinic of diabetes in Tokyo. The diabetic patients with a history of liver disease, kidney disease, and albuminuria (300 mg / g Cr or more) were excluded. The present study was conducted in accordance with the Declaration of Helsinki, and the protocol was approved by the Ethics Committee of the Japan Women's University (No.188), and we explained the purpose and details of this study to the study subjects, and these patients were enrolled with written informed consent.

Albumin / creatinine ratios were measured using casual urine in the morning based on Diabetes Nephropathy diagnostic criteria defined by the Japan Diabetes Association [11]. The study subjects were divided to two groups: a normal albuminuria group with urinary albumin / creatinine ratio of less than $30 \mathrm{mg} / \mathrm{g} \mathrm{Cr}$ and a microalbuminuria group with $30 \mathrm{mg} / \mathrm{g}$ to $299 \mathrm{mg} / \mathrm{g}$ Cr.

At the time of examination, height, weight, BMI, systolic blood pressure, and diastolic blood pressure were measured, and casual blood sampling was performed. Serum total protein (TP) was measured by biuret method. Creatine kinase (CK), aspartate amino transaminase (AST), alanine amino transaminase (ALT), lactate dehydrogenase (LD), alkaline phosphatase (ALP) and $\gamma$ glutamyl transpeptidase $(\gamma$-GT) were measured by JSCC transferable method. Creatinine (Cre) and uric acid (UA) were measured by enzymatic method. Urea nitrogen (UN) was measured by ureaseGLDH-ultra violet method. Glycated hemoglobin (HbA1c) was measured by latex agglutination method. These were measured in the laboratory at BML INC (Tokyo, Japan). In addition, eGFR was calculated using the following formula (male: $194 \times \mathrm{Cr}-1.094 \times$ age (years) -0.287 , female: male eGFR formula $\times 0.739$ ).

The habitual food status, including dietary energy and nutrients, was investigated by a food frequency questionnaire survey, which is standardized for population-based surveys and nutrition counseling in Japan (a food frequency questionnaire based on food group: FFQg version 3.5, manufactured at the site of the Shikoku University Nutrition Database). The survey form was filled 
out to the dietician in terms of dietary habits for the past 1 to 2 months, amounts of foods per day or per meal, and the number of times to eat in one week. The amount per day or per meal, were expressed by comparing with the "normal" amount of each food. We choose one from "not to eat", "little", "normal", "plenty", with half the normal amount being "slightly" and 1.5 times the normal amount being "plenty". In addition, 0.5 times and 0 times were identified when eating at a frequency of once every 2 weeks and when eating at a frequency of less than once a month, respectively. When they had eaten 1 to 2 times per period, it was decided to 1.5 times.

Each intake of energy, carbohydrate, protein, lipid per day was calculated from the results of FFQg. Energy and protein were converted to data per ideal body weight, and ratios of animal protein and vegetable protein were also calculated. The food group composed of 17 items includes cereals, potatoes, green and yellow vegetables, other vegetables / mushrooms, seaweeds, soybean / soy products, fishes and shellfishes, meats, eggs, milks, fruits, confections, beverages, sugars, seeds, oils and fats, seasoning and spices. They were calculated as intake of grams $(\mathrm{g})$. The self-record questionnaire survey about daily life about occupation, diet, smoking, sleeping, exercise and so on were also conducted.

The results were expressed mean \pm standard deviation (SD). Between the normal group and the microalbuminuria group, we analyzed sex difference, the status of smoking and drinking by chisquare tested. Dietary energy, nutrients, intake of food group, obtained by FFQg, BMI, blood pressure data, and biochemical data between the 2 study groups were compared by Mann-Whitney test. All the statistical analyses were performed by using SPSS statics ver.17.0 (IBM, Inc). A significant difference was defined as $\mathrm{p}<0.05$.

\section{Results}

Table 1 shows the characteristics of the normal group and the microalbuminuria group, and Table 2 shows the results of physiological/ biochemical data. There were no significant differences in age, BMI and other physiological and biochemical data between the two groups. HbA1c levels also were comparable between the normal group and the microalbuminuria group $(7.4 \pm 0.6 \%$ and $7.9 \pm$ $1.4 \%$, respectively). However, the systolic blood pressure tended to be insignificantly higher in the microalbuminuria group than in the normal group ( $140 \pm 17 \mathrm{mmHg}$ vs $128 \pm 14 \mathrm{mmHg}, \mathrm{p}=0.084$ ).

The average daily intake of energy and nutrients in the both groups are shown in Table 3. Energy intake levels were $1708 \pm 403 \mathrm{kcal}$ in the normal group and $1706 \pm 351 \mathrm{kcal}$ in the microalbuminuria group, and there was no significant difference between the two groups. Protein intake levels in the 2 groups were not different $(64.0 \pm 18.3 \mathrm{~g} /$ day in the normal group and $70.1 \pm 16.8 \mathrm{~g} /$ day in the microalbuminuria group), and the animal protein intakes also was not different. In carotenoids, $\beta$ cryptoxanthin was significantly lower in the microalbuminuria group than in the normal group (506.4 $\pm 793.9 \mu \mathrm{g} /$ day vs. $715.3 \pm 500.3 \mu \mathrm{g} / \mathrm{day}, \mathrm{p}<0.05)$. But there were no significant differences between the two groups in the other nutrient intakes. The intake of 17 food groups per day showed that the fruits were significantly lower in the microalbuminuria group than in the normal group $(76.9 \pm 134.1$ g vs. $111.9 \pm 84.5 \mathrm{~g} /$ day, $\mathrm{p}<0.05$ ) (Table 4 ). In addition, soybean / soy products, fishes/ shellfishes, eggs and milk were likely to be relatively abundant in the microalbuminuria group, but there were no significant differences.

Moreover, we examined the relationship between $\beta$-cryptoxanthin intake and urinary albumin / creatinine ratio in the subjects, but there was no significant correlation (Figure 1). 
Table 1. Characteristics of subjects

\begin{tabular}{cccc}
\hline & Total & Normal & Microalbuminuria \\
\hline Number (Male / Female) & $42(29 / 13)$ & $29(19 / 10)$ & $13(10 / 3)$ \\
Age (year) & $67.7 \pm 9.4$ & $69.5 \pm 8.5$ & $63.8 \pm 10.8$ \\
Smoking, n (\%) & & & \\
Current & $9(21.4)$ & $5(17.2)$ & $4(30.8)$ \\
Ex-smoker & $9(21.4)$ & $8(27.6)$ & $1(7.7)$ \\
Never & $24(57.1)$ & $16(55.2)$ & $8(61.5)$ \\
Current & & & $2(15.4)$ \\
Non-drinker & $9(21.4)$ & $7(24.1)$ & $11(84.6)$ \\
Drinking alcohol, n $(\%)$ & $33(78.6)$ & $22(75.9)$ & $3(23.1)$ \\
Medication, n (\%) & & & $1(7.7)$ \\
Statin & $8(19.0)$ & $5(17.2)$ & $5(38.5)$ \\
Biguanide & $9(21.4)$ & $8(27.6)$ & $6(46.2)$ \\
DPP-4 inhibitor & $14(33.3)$ & $9(31.0)$ & $2(15.4)$ \\
Sulfonylurea & $20(47.6)$ & $14(48.3)$ & $1(7.7)$ \\
$\alpha$-GI & $10(23.8)$ & $8(27.6)$ & $140 \pm 17$ \\
Diastolic blood pressure $(\mathrm{mmHg})$ & $1(2.4)$ & $0(0.0)$ & $128 \pm 14$ \\
Mass Index (kg/m $\left.{ }^{2}\right)$ & $22.6 \pm 2.9$ & $73 \pm 11$ & \\
\hline
\end{tabular}

The data was expressed as mean \pm standard deviation.

n: number, ARB: angiotensin II receptor blocker, DPP-4: Dipeptidyl peptidase-4, $\alpha$-GI: $\alpha$-glucosidase inhibitor

Table 2. Biological data of the normal group and the microalbuminuria

\begin{tabular}{crrrrrr}
\hline & \multicolumn{2}{c}{ Normal $(\mathrm{n}=29)$} & \multicolumn{2}{c}{ Microalbuminuria $(\mathrm{n}=13)$} \\
\hline TP (g/dL) & 7.2 & \pm & 0.4 & 7.4 & \pm & 0.3 \\
Urea albumin / Creatinine (mg/Cr) & 16.7 & \pm & 26.4 & 74.4 & \pm & $40.3^{* * *}$ \\
Alb (g/dL) & 4.4 & \pm & 0.3 & 4.4 & \pm & 0.3 \\
CK (U/L) & 103.6 & \pm & 61.5 & 84.3 & \pm & 35.2 \\
AST (U/L) & 23 & \pm & 9 & 21 & \pm & 6 \\
ALT (U/L) & 20 & \pm & 10 & 21 & \pm & 6 \\
LD (U/L) & 196 & \pm & 29 & 200 & \pm & 29 \\
ALP (U/L) & 238 & \pm & 77 & 246 & \pm & 85 \\
f-GT (U/L) & 45 & \pm & 98 & 44 & \pm & 37 \\
UN (mg/dL) & 16.4 & \pm & 3.9 & 16.8 & \pm & 3.3 \\
Cre (mg/dL) & 0.75 & \pm & 0.16 & 0.74 & \pm & 0.19 \\
UA (mg/dL) & 5.0 & \pm & 1.1 & 5.6 & \pm & 1.6 \\
eGFR (mL/min/1.73 m $\left.{ }^{2}\right)$ & 74.5 & \pm & 14.0 & 81.0 & \pm & 17.0 \\
HbA1c (\%) & 7.4 & \pm & 0.6 & 7.9 & \pm & 1.4
\end{tabular}

The data was expressed as mean \pm standard deviation, ${ }^{* * *} ; \mathrm{p}<0.0001$. 
TP; Serum total protein, CK; Creatine kinase, AST; aspartate amino transaminase, ALT; alanine amino transaminase, LD; lactate dehydrogenase, ALP; alkaline phosphatase, $\gamma$-GT; $\gamma$ - glutamyl transpeptidase, UN; Urea nitrogen, Cre; Creatinine, UA; uric acid, eGFR; estimated glomerular filtration rate, $\mathrm{HbA1c}$; Glycated hemoglobin.

Table 3. Nutrition assessed by food frequency questionnaire (FFQg)

\begin{tabular}{lrllrll}
\hline & \multicolumn{3}{c}{ Normal $(\mathrm{n}=29)$} & Microalbuminuria $(\mathrm{n}=13)$ \\
\hline Energy (kcal/day) & 1708 & \pm & 403 & 1706 & \pm & 351 \\
Protein (g/day) & 64.0 & \pm & 18.3 & 70.1 & \pm & 16.8 \\
Animal protein (g/day) & 31.8 & \pm & 12.7 & 36.9 & \pm & 14.4 \\
Fat (g/day) & 52.7 & \pm & 18.9 & 54.8 & \pm & 15.3 \\
Carbohydrate (g/day) & 226.7 & \pm & 44.1 & 221.6 & \pm & 56.3 \\
Sodium (mg/day) & 3332 & \pm & 1320 & 3226 & \pm & 1514 \\
Potassium (mg/day) & 2389 & \pm & 723 & 2545 & \pm & 885 \\
Calcium (mg/day) & 652 & \pm & 253 & 743 & \pm & 353 \\
Magnesium (mg/day) & 260 & \pm & 82 & 280 & \pm & 82 \\
Iron (mg/day) & 7.5 & \pm & 2.5 & 8.3 & \pm & 2.4 \\
Retinol ( $\mu$ g/day) & 171 & \pm & 74 & 219 & \pm & 107 \\
$\alpha$-Carotene ( $\mu$ g/day) & 621 & \pm & 353 & 721 & \pm & 520 \\
$\beta$-Carotene ( $\mu$ g/day) & 3805 & \pm & 2070 & 4367 & \pm & 3077 \\
$\beta$-Cryptoxanthin ( $\mu$ g/day) & 715 & \pm & 500 & 506 & \pm & $794 *$ \\
$\alpha$-Tocopherol (mg/day) & 6.1 & \pm & 1.9 & 6.2 & \pm & 2.2 \\
Vitamin C (mg/day) & 107 & \pm & 45 & 98 & \pm & 73 \\
Cholesterol (mg/day) & 260 & \pm & 110 & 344 & \pm & 191 \\
Total fiber (g/day) & 14.8 & \pm & 4.8 & 14.5 & \pm & 5.9 \\
Salt intake (g/day) & 8.4 & \pm & 3.3 & 8.2 & \pm & 3.8 \\
\hline
\end{tabular}

The data was expressed as mean \pm standard deviation, ${ }^{*} ; \mathrm{p}<0.05$

Table 4. Intake volume of food item by food frequency questionnaire (FFQg)

\begin{tabular}{|c|c|c|c|c|c|}
\hline \multirow{2}{*}{$\begin{array}{l}\text { Food item } \\
\text { Grain }(\mathrm{g})\end{array}$} & \multicolumn{3}{|c|}{ Normal $(n=29)$} & \multicolumn{2}{|c|}{ Microalbuminuria $(\mathrm{n}=13)$} \\
\hline & 320.9 & \pm & 80.8 & 295.0 & \pm 113.9 \\
\hline Potatoes (g) & 40.7 & \pm & 35.1 & 34.0 & \pm 35.3 \\
\hline Green vegetables (g) & 89.1 & \pm & 53.2 & 103.4 & \pm 76.7 \\
\hline Other vegetables and Fungi (g) & 181.4 & \pm & 85.9 & 169.7 & \pm 161.2 \\
\hline Sea weeds (g) & 5.9 & \pm & 4.3 & 8.1 & \pm 5.8 \\
\hline Soy and soy products (g) & 92.5 & \pm & 76.1 & 102.8 & \pm 44.4 \\
\hline Fishes (g) & 65.3 & \pm & 36.2 & 77.3 & \pm 42.0 \\
\hline Meats (g) & 49.0 & \pm & 39.5 & 48.5 & \pm 27.0 \\
\hline Eggs (g) & 23.2 & \pm & 19.2 & 38.5 & \pm 40.6 \\
\hline Milk and milk products (g) & 172.5 & \pm & 112.8 & 200.1 & \pm 225.7 \\
\hline Fruits (g) & 111.9 & \pm & 84.5 & 76.9 & $\pm 134.1^{*}$ \\
\hline Sweets and snacks (g) & 42.6 & \pm & 39.5 & 75.7 & \pm 77.7 \\
\hline Alcohol and beverages (g) & 143.0 & \pm & 271.0 & 163.1 & \pm 197.9 \\
\hline
\end{tabular}




\begin{tabular}{lrllrl}
\hline Sugar (g) & 7.5 & \pm & 7.0 & 7.2 & \pm 5.8 \\
Nuts and sesame (g) & 4.8 & \pm & 10.2 & 1.9 & \pm 3.6 \\
Oil (g) & 11.9 & \pm & 10.9 & 8.0 & \pm 5.4 \\
Seasoning and spices (g) & 22.7 & \pm & 13.4 & 19.8 & \pm 10.3 \\
\hline
\end{tabular}

The data was expressed as mean \pm standard deviation, *; $\mathrm{p}<0.05$

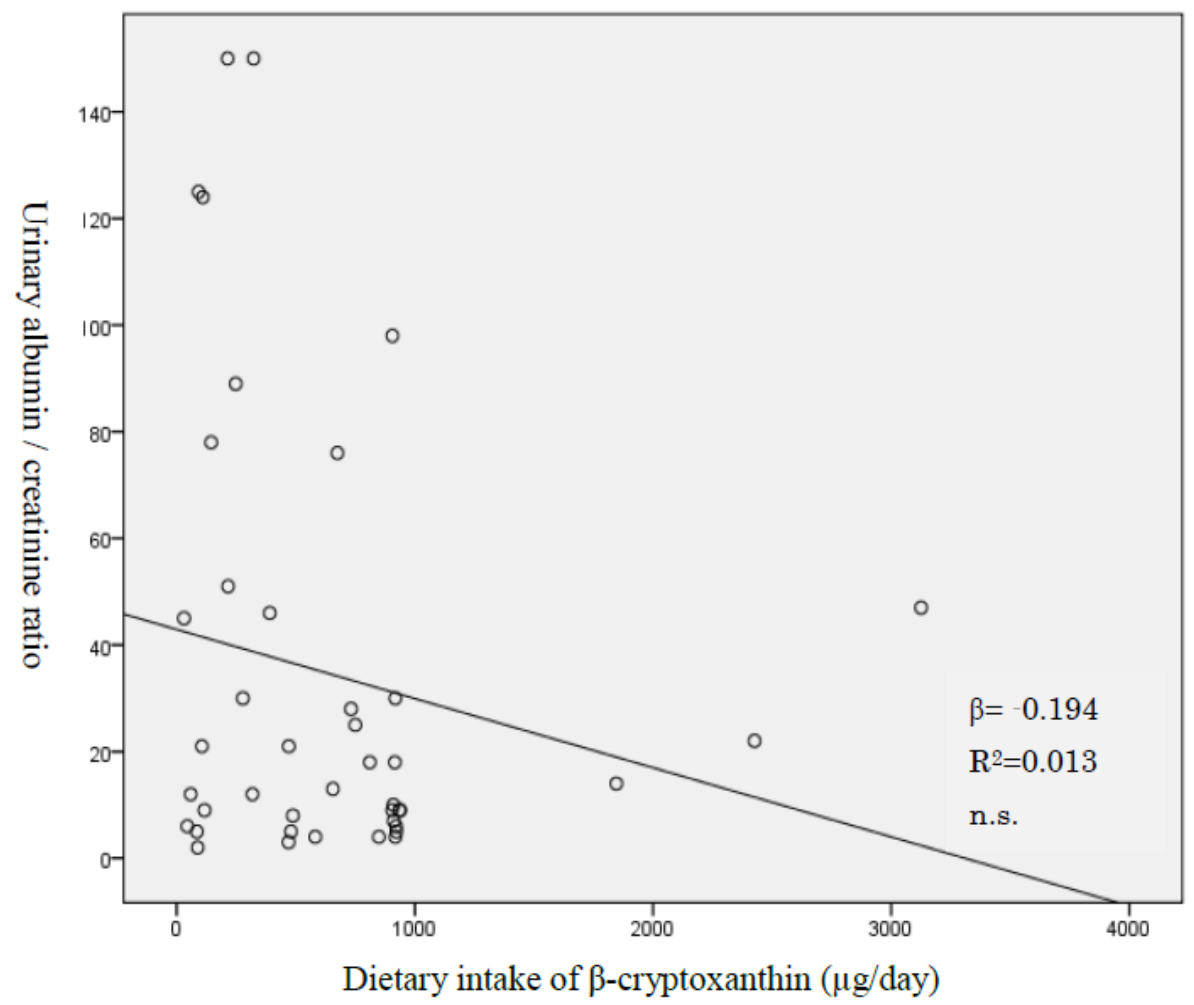

Figure 1. Simgle regression analysis between urinary albumin / creatinine ratio and dietary intake of $\beta$-cryptoxanthin.

$\beta$ : Standardize regression coefficient, $\mathrm{R}^{2}$ : Adjusted coefficient of determination

\section{Discussion}

The relationships between microalbuminuria and meals/ nutrients have been reported in nondiabetic subjects [12], in patients with type 1 diabetes and with type 2 diabetes patients [13,14], but few studies about Japanese type 2 diabetes have been reported so far. Therefore, in this study, we examined the habitual dietary intakes of foods in Japanese type 2 diabetic patients, and those intakes was compared between the normal group and the microalbuminuria group to clarify nutrients and foods affecting albuminuria. As a result, in the physiological and biochemical data, there were no significant differences between the two groups. This is consistent with previous studies $[15,16]$ that control of plasma glucose and blood pressure reduces urinary albumin excretion, and the strict management of plasma glucose and blood pressure is considered important for preventing the development of urinary albumin excretion.

Among the dietary energy and nutrient intakes obtained by FFQ, the intake of $\beta$-cryptoxanthin was significantly lower in the microalbuminuria group than in the normal group $(p<0.05)$. Studies on the relationship between microalbuminuria and dietary intake in healthy subjects have shown that 
there is a negative correlation between $\beta$-cryptoxanthin intake and microalbuminuria in women, and it was reported that the meals having large amount of $\beta$-cryptoxanthin may prevent microalbuminuria [17]. $\beta$-cryptoxanthin is a carotenoid contained in many citrus fruits such as mandarin or oyster, loquat, red pepper and so on. Carotenoids exert strong erasing activity against singlet oxygen and lipid peroxidation. $\mathrm{Ni}$ et al [18] have found that $\beta$-cryptoxanthin administration attenuated insulin resistance and suppressed excessive lipid accumulation, lipid peroxidation, and fibrosis progression, in conjunction with an M2-dominant shift in macrophages/ Kupffer cells in liver of mice fed high-cholesterol and high-fat diet.

It is known that singlet oxygen is generated in the functional process of neutrophils and macrophages which phagocytose and endocytose oxidative denaturation products of bacteria and low-density lipoprotein (LDL) in the body, which is the cause of inflammation and arteriosclerosis [19]. Guessous et al. [20] suggest that high-sensitive $C$ reactive protein (Hs-CRP), a marker of inflammation, may be an independent predictor of renal function decrease, and Rowley et al. [21] have reported that the CRP levels were inversely related to serum $\beta$-cryptoxanthin concentrations.

In this study, the serum $\beta$-cryptoxanthin concentrations of the subjects are unknown, but in patients with diabetic nephropathy, sustaining hyperglycemia and the small amounts of antioxidants in the blood could progress inflammation and arteriosclerosis, possibly leading to a decrease in glomerular function.

$\beta$-cryptoxanthin has been reported to be distributed and stored in kidney tissue in addition to liver and lung after absorbed [22]. The orange juice containing $\beta$-cryptoxanthin inhibits the accumulation of macrophage cells in the kidney, and it acts on the upstream of the nephritis, resulting in the alleviation of morphological abnormalities of basement membrane thickening and podocyte as reported previously [23].

Several investigators have reported that serum carotenoids [24] and plasma concentrations of lycopene, $\beta$-carotene, $\alpha$-carotene and cryptoxanthin [25] were significantly lower in patients with microalbuminuria. $\beta$-cryptoxanthin intake was inversely associated with microalbuminuria [17], while it was not correlated with urinary albumin/ creatinine ratio in the present study. $\beta$ cryptoxanthin concentrations in the blood are more likely to be higher in women than in men even though $\beta$-cryptoxanthin intake is the same between men and women, but habits of smoking and drinking, and obesity are known to reduce concentrations of blood $\beta$-cryptoxanthin [26-29]. In the subjects of this study, $31.0 \%$ were women, and 9 (21.4\%) with smoking, 15 (35.7\%) with drinking, 8 $(19.0 \%)$ with obesity were included, but no differences were found in these factors. Therefore, it would be needed to investigate the relation between $\beta$-cryptoxanthin concentrations in blood and $\beta$ cryptoxanthin intake.

In the food group, amounts of fruits were significantly lower in the microalbuminuria group than in the normal group $(\mathrm{p}<0.05)$. Previous studies have reported that the intake of fruits was associated with microalbuminuria, and in healthy volunteers who have low fruits, urinary albumin excretion is lower as comparted with the subjects with high intake of fruits [12]. Women with type 2 diabetes who eat more than 3 servings of fruits per week have a lower risk of developing chronic kidney disease as compared with the low frequent servings [30]. Furthermore, it was reported that serum CRP levels were lower in highly frequent intakes of fruits and vegetables [31], and in subjects with higher levels of serum CRP, serum concentrations of dietary antioxidant lycopene, $\beta$-carotene, cryptoxanthin and retinol were lower [21]. As these mechanisms, antioxidant components such as carotenoids, vitamins $\mathrm{C}$, vitamin $\mathrm{E}$, and flavonoids contained in fruits and vegetables are considered to have anti-inflammatory effects [31].

In addition, Nettleton et al. [12] reported that low-fat dairy foods and a diet rich in whole grains reduce urinary albumin excretion in healthy people. In this study, we have not investigated these detailed contents of foods. Therefore, it would be necessary to investigate the types of foods to be consumed.

The whole protein and animal protein intake were not significantly different between the two groups, but these were likely to be higher in the microalbuminuria. In a previous study, it has been 
reported that the high intakes of protein and animal protein are related to the presence of microalbuminuria [14]. In an intervention study [32], the protein-restricted diet $(0.8 \mathrm{~g} / \mathrm{kg})$ on early nephropathy in Japanese type 2 diabetic patients significantly decreased microalbuminuria. It was suggested that the intake of protein may affect urinary albumin excretion. The present study was not able to exactly evaluate the protein intake of participants because we used the method of food frequency survey. Moreover, the target population size was small, and consequently the variation in protein intake would be ranged widely. Therefore, no difference in protein intake between the 2 groups were found, and it is unknown whether considering the type of protein consumed can reduce the urinary albumin excretion in diabetic patients.

As a limitation of the present study, the sample size is small as described above, and the possibility of bias on the present results cannot be denied. In addition, because this study is a crosssectional survey on the nutrient and dietary factors influencing urinary albumin excretion, the causal relationships between these factors and urinary albumin excretion cannot be determined. In the future, it is necessary to investigate the intervention studies whether the intake of foods containing many antioxidant components including $\beta$-cryptoxanthin reduces urinary albumin excretion in patients with type 2 diabetes.

\section{Conclusions}

In the present study, dietary intakes of $\beta$-cryptoxanthin and fruits were lower in type 2 diabetic patients with microalbuminuria. These results suggest that fruits and foods rich in $\beta$-cryptoxanthin would make it possible to prevent diabetic nephropathy progression, but prospective controlled studies are needed to confirm these findings.

Author Contributions: Conceptualization, S.M. and Y.S.; Methodology, S.M.; Formal analysis, S.M.; Investigation, S.M.; Resources, Y.S.; Data curation, S.M.; Writing-original draft preparation, S.M.; Writingreview and editing, S.M., Y.S, and H.Y.; Supervision, H.Y.; Project administration, Y.S.

Funding: Research funds were provided in part by the Jikei University Research Fund from the Jikei University School of Medicine and Grant-in-Aid for Scientific Research (number 17K09560) from Japan Ministry of Education, Culture, Sports, Science, and Technology (Hiroshi Yoshida). The funders had no role in the design of the study; in the collection, analyses, or interpretation of data; in the writing of the manuscript, or in the decision to publish the results.

Acknowledgments: This study was performed by Hana Onodera, Mizuho Komiya, Erika Show and Mio Goto.

Conflicts of Interest: There are no conflicts of interest.

\section{References}

1. Masakane, I.; Taniguchi, M.; Nakai, S.; Tsuchida, K.; Wada, A.; Ogata, S.; Hasegawa, T.; Hamano, T.; Hanafusa, N.; Hoshino, J., et al. An overview of regular dialysis treatment in Japan as of Dec, 31, 2016. Journal of Japanese Society for Dialysis Therapy 2018, 51, 1-51, doi:10.4009/jsdt.51.1.

2. Nagata M, N.T., Kiyohara Y, Murakami Y, Irie F, Sairenchi T, Miura K, Okamura T, Ueshima H; EPOCHJAPAN Research Group. Prediction of cardiovascular disease mortality by proteinuria and reduced kidney function: pooled analysis of 39,000 individuals from 7 cohort studies in Japan. Am J Epidemiol 2013, 1, 111.

3. Nagata, M.; Ninomiya, T.; Kiyohara, Y.; Murakami, Y.; Irie, F.; Sairenchi, T.; Miura, K.; Okamura, T.; Ueshima, H. Prediction of cardiovascular disease mortality by proteinuria and reduced kidney function: pooled analysis of 39,000 individuals from 7 cohort studies in Japan. Am J Epidemiol 2013, 178, 1-11, doi:10.1093/aje/kws447.

4. Masakane, I.; Nakai, S.; Ogata, S.; Kimata, N.; Hanafusa, N.; Hamano, T.; Wakai, K.; Wada, A.; Nitta, K. An Overview of Regular Dialysis Treatment in Japan (As of 31 December 2013). Therapeutic apheresis and dialysis: official peer-reviewed journal of the International Society for Apheresis, the Japanese Society for Apheresis, the Japanese Society for Dialysis Therapy 2015, 19, 540-574, doi:10.1111/1744-9987.12378. 
5. Haneda, M.; Utsunomiya, K.; Koya, D.; Babazono, T.; Moriya, T.; Makino, H.; Kimura, K.; Suzuki, Y.; Wada, T.; Ogawa, S., et al. [Classification of Diabetic Nephropathy 2014]. Nihon Jinzo Gakkai shi 2014, 56, 547552.

6. Araki, S.; Haneda, M.; Koya, D.; Hidaka, H.; Sugimoto, T.; Isono, M.; Isshiki, K.; Chin-Kanasaki, M.; Uzu, T.; Kashiwagi, A. Reduction in microalbuminuria as an integrated indicator for renal and cardiovascular risk reduction in patients with type 2 diabetes. Diabetes 2007, 56, 1727-1730, doi:10.2337/db06-1646.

7. Bouchi, R.; Babazono, T.; Yoshida, N.; Nyumura, I.; Toya, K.; Hayashi, T.; Hanai, K.; Tanaka, N.; Ishii, A.; Iwamoto, Y. Association of albuminuria and reduced estimated glomerular filtration rate with incident stroke and coronary artery disease in patients with type 2 diabetes. Hypertens Res 2010, 33, 1298-1304, doi:10.1038/hr.2010.170.

8. Pedrini, M.T.; Levey, A.S.; Lau, J.; Chalmers, T.C.; Wang, P.H. The effect of dietary protein restriction on the progression of diabetic and nondiabetic renal diseases: a meta-analysis. Annals of internal medicine 1996, 124, 627-632.

9. Pan, Y.; Guo, L.L.; Jin, H.M. Low-protein diet for diabetic nephropathy: a meta-analysis of randomized controlled trials. Am J Clin Nutr 2008, 88, 660-666, doi:10.1093/ajcn/88.3.660.

10. The Japan diabetes society. (2016) Practice guideline for the treatment for diabetes in Japan 2016, N.C., Tokyo; pp48. Practice guideline for the treatment for diabetes in Japan 2016; Nankodo: Tokyo, Japan, 2016.

11. Inomat, S.; Haneda, M.; Moriya, T.; Katayama, S.; Iwamoto, Y.; Sakai, H.; Tomino, Y.; Matsuo, S.; Asano, Y.; Makino, H. [Revised criteria for the early diagnosis of diabetic nephropathy]. Nihon Jinzo Gakkai shi 2005, 47, 767-769.

12. Nettleton, J.A.; Steffen, L.M.; Palmas, W.; Burke, G.L.; Jacobs, D.R., Jr. Associations between microalbuminuria and animal foods, plant foods, and dietary patterns in the Multiethnic Study of Atherosclerosis. Am J Clin Nutr 2008, 87, 1825-1836, doi:10.1093/ajcn/87.6.1825.

13. Engelen, L.; Soedamah-Muthu, S.S.; Geleijnse, J.M.; Toeller, M.; Chaturvedi, N.; Fuller, J.H.; Schalkwijk, C.G.; Stehouwer, C.D. Higher dietary salt intake is associated with microalbuminuria, but not with retinopathy in individuals with type 1 diabetes: the EURODIAB Prospective Complications Study. Diabetologia 2014, 57, 2315-2323, doi:10.1007/s00125-014-3367-9.

14. Almeida, J.C.; Zelmanovitz, T.; Vaz, J.S.; Steemburgo, T.; Perassolo, M.S.; Gross, J.L.; Azevedo, M.J. Sources of protein and polyunsaturated fatty acids of the diet and microalbuminuria in type 2 diabetes mellitus. Journal of the American College of Nutrition 2008, 27, 528-537.

15. Araki, S.; Haneda, M.; Sugimoto, T.; Isono, M.; Isshiki, K.; Kashiwagi, A.; Koya, D. Factors associated with frequent remission of microalbuminuria in patients with type 2 diabetes. Diabetes 2005, 54, 2983-2987.

16. Yamada, T.; Komatsu, M.; Komiya, I.; Miyahara, Y.; Shima, Y.; Matsuzaki, M.; Ishikawa, Y.; Mita, R.; Fujiwara, M.; Furusato, N., et al. Development, progression, and regression of microalbuminuria in Japanese patients with type 2 diabetes under tight glycemic and blood pressure control: the Kashiwa study. Diabetes care 2005, 28, 2733-2738.

17. Sato, M.; Hozawa, A.; Konta, T.; Shao, L.; Otani, K.; Narimatsu, H.; Sasaki, S.; Kato, T.; Kubota, I.; Yamashita, H., et al. Relationship between dietary intake and microalbuminuria: findings from the Takahata study. Clinical and experimental nephrology 2012, 16, 147-155, doi:10.1007/s10157-011-0539-5.

18. Ni, Y.; Nagashimada, M.; Zhan, L.; Nagata, N.; Kobori, M.; Sugiura, M.; Ogawa, K.; Kaneko, S.; Ota, T. Prevention and reversal of lipotoxicity-induced hepatic insulin resistance and steatohepatitis in mice by an antioxidant carotenoid, beta-cryptoxanthin. Endocrinology 2015, 156, 987-999, doi:10.1210/en.2014-1776.

19. Terao J, N.A. Absorption, metabolism and physiological functions of carotenoids. Journal of Japan Oil Chemist's Society 1999, 48, 1075-1085, doi:10.5650/jos1996.48.1075.

20. Guessous, I.; Ponte, B.; Marques-Vidal, P.; Paccaud, F.; Gaspoz, J.M.; Burnier, M.; Waeber, G.; Vollenweider, P.; Bochud, M. Clinical and biological determinants of kidney outcomes in a population-based cohort study. Kidney \& blood pressure research 2014, 39, 74-85, doi:10.1159/000355779.

21. Rowley, K.; Walker, K.Z.; Cohen, J.; Jenkins, A.J.; O'Neal, D.; Su, Q.; Best, J.D.; O'Dea, K. Inflammation and vascular endothelial activation in an Aboriginal population: relationships to coronary disease risk factors and nutritional markers. The Medical journal of Australia 2003, 178, 495-500.

22. Sugiura, M.; Ogawa, K.; Yano, M. Absorption, storage and distribution of beta-cryptoxanthin in rat after chronic administration of Satsuma mandarin (Citrus unshiu MARC.) juice. Biological \& pharmaceutical bulletin 2013, 36, 147-151.

23. Hikita, M.; Motojima, K.; Kamata, S.; Yoshida, T.; Tanaka-Nakadate, S.; Nakadate, K. Protective Efficacy of the Ingestion of Mandarin Orange Containing beta-Cryptoxanthin on Lipopolysaccharide-induced Acute 
Nephritis. Yakugaku zasshi : Journal of the Pharmaceutical Society of Japan 2016, 136, 1031-1040, doi:10.1248/yakushi.15-00284.

24. Ford, E.S.; Giles, W.H.; Mokdad, A.H.; Ajani, U.A. Microalbuminuria and concentrations of antioxidants among US adults. American journal of kidney diseases: the official journal of the National Kidney Foundation 2005, 45, 248-255.

25. Rowley, K.; O'Dea, K.; Su, Q.; Jenkins, A.J.; Best, J.D. Low plasma concentrations of diet-derived antioxidants in association with microalbuminuria in Indigenous Australian populations. Clinical science (London, England: 1979) 2003, 105, 569-575, doi:10.1042/cs20030162.

26. Sugiura, M.; Matsumoto, H.; Kato, M.; Ikoma, Y.; Yano, M.; Nagao, A. Multiple linear regression analysis of the seasonal changes in the serum concentration of beta-cryptoxanthin. Journal of nutritional science and vitaminology 2004, 50, 196-202.

27. Gabriel, H.E.; Liu, Z.; Crott, J.W.; Choi, S.W.; Song, B.C.; Mason, J.B.; Johnson, E.J. A comparison of carotenoids, retinoids, and tocopherols in the serum and buccal mucosa of chronic cigarette smokers versus nonsmokers. Cancer epidemiology, biomarkers \& prevention: a publication of the American Association for Cancer Research, cosponsored by the American Society of Preventive Oncology 2006, 15, 993-999, doi:10.1158/1055-9965.Epi-05-0664.

28. Widome, R.; Jacobs, D.R., Jr.; Hozawa, A.; Sijtsma, F.; Gross, M.; Schreiner, P.J.; Iribarren, C. Passive smoke exposure and circulating carotenoids in the CARDIA study. Annals of nutrition \& metabolism 2010, 56, 113-118, doi:10.1159/000277662.

29. Sugiura, M.; Nakamura, M.; Ogawa, K.; Ikoma, Y.; Matsumoto, H.; Ando, F.; Shimokata, H.; Yano, M. Synergistic interaction of cigarette smoking and alcohol drinking with serum carotenoid concentrations: findings from a middle-aged Japanese population. The British journal of nutrition 2009, 102, 1211-1219, doi:10.1017/s0007114509382124.

30. Dunkler, D.; Dehghan, M.; Teo, K.K.; Heinze, G.; Gao, P.; Kohl, M.; Clase, C.M.; Mann, J.F.; Yusuf, S.; Oberbauer, R. Diet and kidney disease in high-risk individuals with type 2 diabetes mellitus. JAMA internal medicine 2013, 173, 1682-1692, doi:10.1001/jamainternmed.2013.9051.

31. Gao, X.; Bermudez, O.I.; Tucker, K.L. Plasma C-reactive protein and homocysteine concentrations are related to frequent fruit and vegetable intake in Hispanic and non-Hispanic white elders. The Journal of nutrition 2004, 134, 913-918, doi:10.1093/jn/134.4.913.

32. Narita, T.; Koshimura, J.; Meguro, H.; Kitazato, H.; Fujita, H.; Ito, S. Determination of optimal protein contents for a protein restriction diet in type 2 diabetic patients with microalbuminuria. The Tohoku journal of experimental medicine 2001, 193, 45-55. 